\title{
Allemagne: Nouvelle loi sur la psychothérapie avant sa conclusion
}

Peter Schulthess

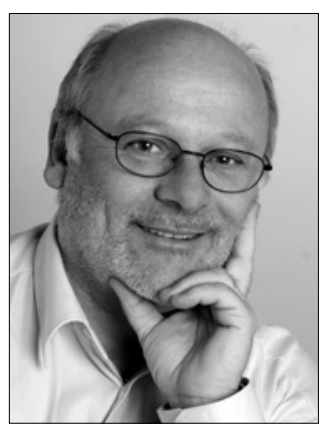

Une révision de la loi sur la psychothérapie (PsyG) est en cours en Allemagne, ce qui est susceptible d'avoir un impact majeur à léchelle européenne. Le précédent règlement, qui exigeait d'abord un diplôme en psychologie afin de pouvoir ensuite poursuivre la formation en psychothérapie, doit être remplacé par une loi prévoyant létude directe en psychothérapie. Il s'agit d'une maitrise scientifique de cinq ans, qui conduit à l'approbation (autorisation de traitement) et permet ainsi l'accès à la profession. Il est divisé en un niveau bachelor de trois ans et un niveau Master de deux ans.

Les chevauchements de contenu entre létude de la psychologie et de la formation continue sont éliminés, ce qui laisse la place à l'inclusion d'autres matières de base relevant d'autres disciplines des sciences sociales et culturelles concernant la psychothérapie, par exemple, comme le suggère létude complémentaire de psychothérapie de la Charte ou la filière universitaire de psychologie psychothérapeutique de l'université du Danube à Krems en coopération avec la Charte. Létude de la psychothérapie est enseignée exclusivement dans les universités et les hautes écoles équivalentes.

Elle combine l'enseignement des connaissances de base et une formation en psychothérapie. Toutes les thérapies fondées sur des preuves scientifiques seront enseignées dans le cours, même celles qui sont proches dêtre considérées comme scientifiquement valables. Le titre professionnel est désormais "psychothérapeute » et remplace les titres de "psychologue psychothérapeute » ou "psychothérapeute pour enfants et adolescents ».

Sur la base de l'approbation d'exercer, la formation axée sur les processus et le groupe d'âge peut être lancée. Le programme comprend également une formation continue en établissement hospitalier ou ambulatoire, organisée conformément à la loi locale. Les services de traitement des psychothérapeutes en formation continue (PiW) sont rémunérés par les compagnies d'assurance maladie. Une fois la formation continue terminée, les psychothérapeutes ont le droit de s'inscrire au registre des médecins et de demander leur admission dans le système d'assurance maladie obligatoire.
Le 3 janvier 2019, le ministère fédéral de la Santé a présenté un projet de loi correspondant, qui a été adopté le 27 février 2019 par le Cabinet fédéral. Il nécessite encore l'approbation du Conseil fédéral. La loi sera promulguée dans le second semestre de 2019. Les premiers cours directs sont prévus pour le semestre d'hiver 2020/21. Pour plus d'informations, veuillez visiter le site Web suivant : https://www.bundesgesundheitsministerium.de/ psychotherapeutenausbildung.html

\section{Commentaire}

Dans toute l'Europe, la formation en psychothérapie gagne du terrain. L'un des pionniers d'une étude directe en psychothérapie est entre autres, l'Université privée Sigmund Freud de Vienne, qui enseigne la psychothérapie en Autriche et dans d'autres pays européens. En Allemagne, d'autres universités et facultés de psychologie suivent désormais ce modèle.

Comme d'habitude, la Suisse devra adapter sa législation à l'Allemagne et à d'autres pays européens avec quelques années de retard. En Allemagne, à cette époque, une loi a été adoptée de manière clairvoyante sur la réglementation de la psychologie et une autre sur la régulation de la psychothérapie. À loccasion du travail législatif, l'ASP a également exigé une loi distincte sur la psychologie et une loi sur la psychothérapie pour réglementer la profession de psychothérapeute en tant que profession indépendante. Cependant, la Suisse ne l'a pas souhaité et a préféré définir la psychothérapie comme une profession de psychologie dans une seule loi, comme par le passé, la médecine définissait la psychothérapie comme une profession médicale.

C'était trop séduisant pour les autorités et pour les politiciens de créer un lien logique entre la psychologie et la psychothérapie d'une manière simplifiée, analogue à la combinaison médecin-psychiatre/psychothérapeute pour mettre fin à la discussion sur la psychothérapie en tant que profession scientifique indépendante accessible à partir d'autres diplômes sociaux. Il n’a pas été pris en compte que l'examen d'état médical correspond à une admission professionnelle dans l'activité médicale. Létude médicale 
est donc une formation professionnelle dans un corps médical, suivie d'une spécialisation dans une branche de la médecine qui donne le titre de médecin spécialiste. Létude de la psychologie, y compris la psychologie clinique, est différente : Il ne constitue pas une formation professionnelle dans une profession médicale pouvant être exercée après l'obtention du diplôme. La formation professionnelle réelle en tant que psychothérapeute se déroule seulement dans la formation continue. À cet égard, l'argumentation des représentants de la psychologie avec cette analogie a toujours été fausse, mais a eu de l'impact.

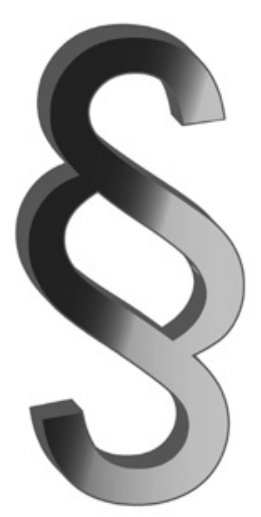

Le nouveau modèle allemand remplace désormais la psychothérapie du devoir d'étudier la psychologie et la réglemente en tant que profession indépendante pouvant être apprise dans une étude directe. Si le modèle de formation d'une étude directe en psychothérapie, qui représente en même temps une formation professionnelle en psychothérapie, est également appliqué dans d'autres pays, la psychothérapie devient alors une profession scientifique indépendante, qui peut être apprise dès le départ et ne nécessite pas au préalable un diplôme en psychologie.

La réglementation de la psychothérapie en Suisse doit alors probablement être retirée du PsyG et être cependant réglementée par une loi distincte.
Cela aurait pu être plus facile. L'ASP et la Charte étaient sans doute en avance sur le temps.

La période de formation et donc les coûts de formation sont sensiblement réduits avec ce modèle. Le projet de loi allemand prévoit qu'avec un diplôme de maîtrise, l'approbation peut être demandée (analogue à l'examen d'État en médecine), et celui qui passe, est autorisé à exercer la profession de psychothérapeute dès lors. Une spécialisation dans une direction thérapeutique spécifique (spécialisation) ou dans un groupe d’âge spécifique ou un groupe de troubles spécifique a ensuite lieu et conduit au titre de psychothérapeute spécialisé(e).

Les instituts privés de formation continue devront rechercher une coopération avec les universités en vue de l'acquisition de la formation psychothérapeutique s'ils souhaitent continuer à participer à la formation en psychothérapie. Ou ils se concentrent sur les spécialisations de troisième cycle des psychothérapeutes dans certaines procédures ou sur des sujets spécifiques. En outre, l'importance de la recherche apparaît clairement, ce qui peut prouver la nature fondée sur des preuves d’orientations thérapeutiques apprises.

Je suis convaincue que toutes les associations et les instituts de psychologie soutiendront ce développement et que de tels cours directs de psychothérapie seront également offerts dans les universités suisses dès qu'un changement correspondant dans la loi deviendra apparent. Mais là aussi, bien sûr, des incertitudes politiques sont présentes.

Dans toute l'Europe, on peut voir que l'academisation de la formation de psychothérapie progresse et que la profession de psychothérapeute sera réglementée comme une profession indépendante dans d'autres pays également et les programmes d'études directs remplaceront le modèle actuel de formation. L'harmonisation européenne des régimes de psychothérapie n'est qu'une question de temps.

\footnotetext{
Peter Schulthess est membre du Conseil d'administration de l'ASP et le représente avec Gabriela Rüttimann au sein de l'EAP. Dans ce rôle, il suit le développement international de la psychothérapie.
} 